\title{
The Association of Big Flares and Coronal Mass Ejections: What is the Role of Magnetic Helicity?
}

\author{
A. Nindos ${ }^{1}$ and M.D. Andrews ${ }^{2}$ \\ ${ }^{1}$ Section of Astrogeophysics, Physics Department, University of Ioannina, Ioannina GR-45110, \\ Greece \\ email: anindos@cc.uoi.gr \\ ${ }^{2}$ Computational Physics Inc, NRL, Code 7660, Washington DC 20375, USA \\ email:michael.andrews@nrl.navy.mil
}

\begin{abstract}
In a recent study Andrews found that approximately $40 \%$ of M-class flares between 1996 and 1999, classified according to GOES X-ray flux, are not associated with Coronal Mass Ejections (CMEs). Using 133 events from his dataset for which suitable photospheric magnetograms and coronal images were available, we studied the pre-flare coronal helicity of the active regions that produced big flares. The coronal magnetic field of 78 active regions was modeled under the "constant $\alpha$ " linear force-free field assumption. We find that in a statistical sense the pre-flare value of $\alpha$ and coronal helicity of the active regions producing big flares that do not have associated CMEs is smaller than the coronal helicity of those producing CME-associated big flares. A further argument supporting this conclusion is that for the active regions whose coronal magnetic field deviates from the force-free model, the change of the coronal sign of $\alpha$ within an active region is twice more likely to occur when the active region is about to produce a confined flare than a CME-associated flare. Our study indicates that the amount of the stored pre-flare coronal helicity may determine whether a big flare will be eruptive or confined.
\end{abstract}

Keywords. solar-terrestrial relations - Sun: activity -Sun: flares - Sun: coronal mass ejections -Sun: magnetic fields -Sun: corona

\section{Introduction}

Recently, Andrews (2003) considered the complete list of the X- and M-class GOES soft X-ray flares observed during the years 1996-1999. He identified possible CME candidates for the 229 flares of his list with good LASCO coverage and concluded that $40 \%$ of the M-class flares do not have associated CMEs. The probability of finding a CME candidate did not depend on the solar location of the flare which supports the conclusion that the lack of observed CMEs was not an observational selection effect. In this paper we shall try to understand why some M-class flares do have associated CMEs while other M-class flares do not. Our data set consists of the events studied by Andrews (2003). For such task, one needs to study in detail the properties of the active regions (ARs) which produce the big flares. Here we shall compute the coronal magnetic helicity of the corresponding active regions prior to the flare onset. Our study will demonstrate that the coronal magnetic helicity of the ARs plays an important role concerning the association (or the absence thereof) of big flares with CMEs.

\section{Coronal Magnetic Helicity}

The magnetic helicity of a field $\mathbf{B}$ within a volume $V$ is defined as $H_{m}=\int_{V} \mathbf{A} \cdot \mathbf{B} d V$ where $\mathbf{A}$ is the magnetic vector potential. The magnetic helicity is physically meaningful only when B is fully contained inside $V$. When this condition is not satisfied (for example in the solar atmosphere), we define a gauge-independent relative magnetic helicity (hereafter referred to as 
Table 1. Active Regions and LFFF Extrapolations

\begin{tabular}{lccc}
\hline Extrapolation & $\begin{array}{c}\text { Active Regions } \\
\text { (Flare-CME) }\end{array}$ & $\begin{array}{c}\text { Active Regions } \\
\text { (Flare, no CME) }\end{array}$ & $\begin{array}{c}\text { Total } \\
\text { Number }\end{array}$ \\
\hline Acceptable & 47 & 31 & 78 \\
Both signs of $\alpha$ & 15 & 25 & 40 \\
Uniform $\alpha$ sign, & 10 & 5 & 15 \\
large deviation & 10 & 5 \\
\hline
\end{tabular}

helicity) of $\mathbf{B}$ with respect to the helicity of a reference field $\mathbf{B}_{\mathbf{p}}$ having the same distribution of vertical magnetic flux on the surface $S$ surrounding $V: H=\int_{V} \mathbf{A} \cdot \mathbf{B} d V-\int_{V} \mathbf{A}_{\mathbf{p}} \cdot \mathbf{B}_{\mathbf{p}} d V$. Being a potential field it is a convenient choice for $\mathbf{B}_{\mathbf{p}}$. The quantity $\mathbf{A}_{\mathbf{p}}$ is the corresponding vector potential satisfying $\nabla \cdot \mathbf{A}_{\mathbf{p}}=0$ and being horizontal on $S$. Then the term $\int_{V} \mathbf{A}_{\mathbf{p}} \cdot \mathbf{B}_{\mathbf{p}} d V$ vanishes (Berger 1988).

In an open volume like the solar atmosphere, helicity can change either because of the emergence of new twisted field lines that cross the photospheric surface or/and by shearing motions on the photospheric surface. Such motions include differential rotation and/or transient flows. On the other hand when a CME is launched, it carries away part of the helicity of its source magnetic field. Demoulin et al. (2002) and Green et al. (2002) developed a method to compute the coronal helicity $H_{c}$ of ARs. A photospheric magnetogram is used as boundary condition for linear force-free field (lfff) magnetic extrapolations $(\nabla \times \mathbf{B}=\alpha \mathbf{B}$ with $\alpha$ being constant over the AR). The extrapolated field lines are fitted with the AR's coronal loops. The value of $\alpha$ giving the best overall fit between the models and observations is adopted for the computation of the coronal helicity. Then one follows Berger (1985) and after linearizing the derived expression in order to avoid helicity enhancements close to the resonance values, the resulting coronal helicity is

$$
H_{c}=2 \alpha \sum_{n_{x}=1}^{N_{x}} \sum_{n_{y}=1}^{N_{y}} \frac{\left|\tilde{B}_{n_{x}, n_{y}}^{2}\right|}{\left(k_{x}^{2}+k_{y}^{2}\right)^{3 / 2}}
$$

where $\tilde{B}_{n_{x}, n_{y}}$ is the magnetic field's Fourier amplitude of the $\left(n_{x}, n_{y}\right)$ harmonic, $k_{x}=2 \pi n_{x} / L$, $k_{y}=2 \pi n_{y} / L$ with $L$ being the horizontal extension of the computation box used for the forcefree field extrapolations.

\section{Results}

From the 229 flares studied by Andrews (2003), we select those which originate from ARs located within $\pm 50^{\circ}$ from the central meridian at the time of the flare. For the time interval which starts 1.5 hours prior to the flare start time (as defined in the GOES catalogs) we require the availability of at least one MDI magnetogram and EIT $195 \AA$ images obtained with cadence higher than $25 \mathrm{~min}$. The above selection criteria yield 133 events for further analysis. For each case we use the MDI magnetogram taken 25 min prior to the flare start time as boundary condition for linear force-free field extrapolations. This is possible when 1-min-cadence MDI magnetograms are available. When they are not available, we create a magnetogram for the time we need, taking into account the solar rotation and interpolating the two magnetograms obtained closest to the desired time.

For each event, the extrapolated field lines are fitted with the corresponding AR's EIT coronal loops. We determine the best value of $\alpha$ iteratively following basically the procedure developed by Green et al. (2002). The interested reader is referred to their $\S 2.6$ for details. Here, we summarize the technique briefly: (1) We calculate the mean distance $d_{\text {mean }}$ between a given EIT loop and the computed field lines resulted from a given $\alpha$. (2) Through successive steps we select the value of $\alpha$ which gives the lowest $d_{\text {mean }}$ for the loop. (3) The same procedure is repeated for all loops appearing in the EIT image. The value of $\alpha$ giving the best overall 

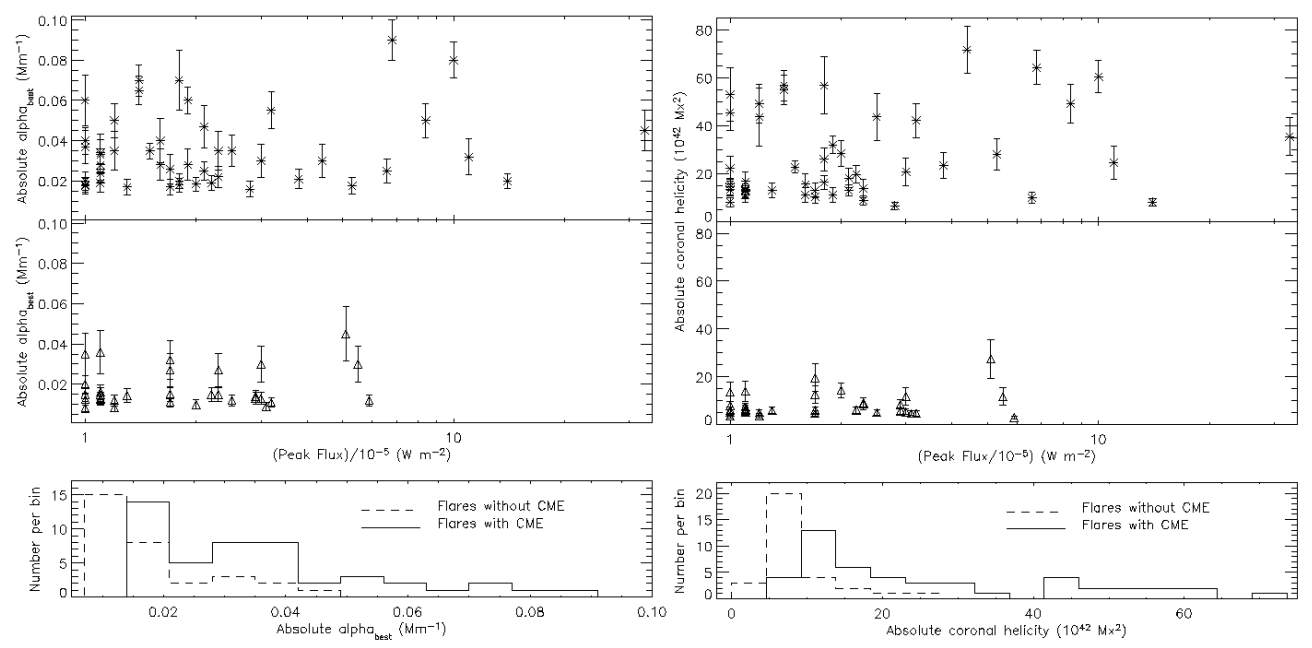

Figure 1. Left column, top: Scatter plot of the pre-flare absolute values of $\alpha_{\text {best }}$ as a function of the flare's peak X-ray flux for the ARs producing CME-associated flares. Left column, middle: Same as top panel, but for the ARs producing flares that do not have associated CMEs. See text for details concerning the error bars. Left column, bottom: Histograms of the values of $\alpha_{\text {best }}$ appearing in the top and middle panels. The solid line represents the histogram of $\alpha_{\text {best }}$ of the ARs which give CME-associated flares while the dashed line is the histogram of $\alpha_{\text {best }}$ of the ARs which produce flares that do not have CMEs. Right column: The absolute coronal helicity of the 78 ARs appearing in the left panel. The format is identical to the format of the left column.

fit between the models and observations $\left(\alpha_{\text {best }}\right)$ is the one which minimizes $\left\langle d_{\text {mean }}\right\rangle$. The derived $\alpha_{\text {best }}$ is considered satisfactory and used in the subsequent analysis if two conditions are met: (1) the derived values of $\alpha$ for individual loops should all have the same sign and (2) $<d_{\text {mean }}>\leqslant 1.9 \mathrm{Mm}$ which is close to the pixel size of the high-resolution EIT images. The above criteria have been implemented because the constant value of $\alpha$ above an active region is a simplification. The values of $\alpha_{\text {best }}$ which survive the two criteria are associated with mean deviations that never exceed $25 \%-30 \%$ of the corresponding $\alpha_{b e s t}$. In Table 1 we give the number of ARs which satisfy both conditions, the number of ARs that do not pass the first condition, and the number of ARs that pass the first condition but do not satisfy the second. In Table 1 we give separately the numbers of ARs producing CME-associated flares and the numbers of ARs producing flares that do not have CMEs.

In fig. 1 (left column) we show the absolute values of $\alpha_{\text {best }}$ of the 78 ARs which passed our two conditions as a function of flare's peak flux. Each error bar denotes the mean deviation to the value of $\alpha_{\text {best }}$ over the AR. Also in the left column of fig. 1 we give the histograms of $\alpha_{\text {best }}$. A visual inspection of the scatter plots indicates that there is a weak correlation between the values of $\alpha_{\text {best }}$ and the corresponding X-ray peak flux; the correlation coefficient is 0.21 . Similar small correlation coefficients have been derived between the values of $\alpha_{\text {best }}$ and the total X-ray flux and duration of the flares (the corresponding scatter plots are not given for the sake of brevity). The average of all values of $\alpha_{b e s t}$ of fig. 1 is $0.028 \pm 0.017 \mathrm{Mm}^{-1}$. This is about a factor of 4 larger than the average photospheric $\alpha_{b e s t}$ derived by Pevtsov, Canfield \& Metcalf (1995) who studied 69 diverse ARs with varying level of flare activity. The large difference between the two studies is due to selection effects: our sample consists of ARs observed a few minutes before powerful flares. In the right column of fig. 1 we give the scatter plots and histograms of the absolute coronal helicity, $H_{c}$, using the values of $\alpha_{b e s t}$ and eq. (2.1). The average of all values of $H_{c}$ is $(19.5 \pm 17.0) \times 10^{42} \mathrm{Mx}^{2}$.

Fig. 1 shows that several ARs which give big flares without CMEs have smaller values of $\alpha_{\text {best }}$ and $H_{c}$ than those producing CME-associated flares. This result shows better in the histograms and it is statistically significant. We have computed the average $\alpha_{b e s t}$ and $H_{c}$ separately for 
the ARs which give flares that do not have CMEs and for the ARs which give CME-associated flares. We find: $\left\langle\alpha_{\text {nocme }}>=0.018 \pm 0.010 \mathrm{Mm}^{-1},<\alpha_{\text {cme }}>=0.035 \pm 0.018 \mathrm{Mm}^{-1}\right.$ and $<H_{\text {nocme }}>=(8.3 \pm 5.2) \times 10^{42} \mathrm{Mx}^{2},<H_{\text {cme }}>=(26.8 \pm 18.1) \times 10^{42} \mathrm{Mx}^{2}$. From the scatter plots, and taking into account the error bars, we find that $45 \%$ of the events without CME come from ARs with smaller values of $\alpha_{\text {best }}$ than the values of $\alpha_{\text {best }}$ of each and every AR which gave CME-associated flare. A similar percentage (52\%) has been found for the coronal helicities of the ARs without CMEs with respect to the coronal helicities of all ARs producing CME-associated events. The analysis of our results for the $n=78$ ARs appearing in fig. 1 shows that the ARs with $\alpha_{\text {best }}>0.02 \mathrm{Mm}^{-1}$ are a factor of 2.25 more likely to produce flare with CME than the ARs with $\alpha_{\text {best }} \leqslant 0.02 \mathrm{Mm}^{-1}$. We have used the $\phi$ coefficient for evaluation of statistical significance of the above result. This coefficient is related to chi-square values through $X^{2}=n \phi^{2}$, which can be compared to tabulated chi-square values with one degree of freedom. For our dataset we find $X^{2}=16.4$, which means that the null hypothesis (i.e. that there is no association between the initiation of CME-associated flare and whether the AR's $\alpha_{\text {best }}$ is bigger or smaller than $0.02 \mathrm{Mm}^{-1}$ ) can be rejected at better than the $99.5 \%$ confidence level. By the same measures, the ARs with $H_{c}>15 \times 10^{42} \mathrm{Mx}^{2}$ are a factor of 2.4 more likely to produce flare with CME than the ARs with $H_{c} \leqslant 15 \times 10^{42} \mathrm{Mx}^{2}$. Here we obtain $X^{2}=23.2$ and again the null hypothesis can be rejected at better than the $99.5 \%$ confidence level.

The EIT images show low-lying, relatively cool loops. In order to prove that our results are accurate, our best-fit magnetic extrapolations should be checked against Yohkoh SXT and TRACE images. From the 78 ARs appearing in fig. 1, 52 of them have been observed simultaneously by EIT and SXT and 7 of them by EIT and TRACE. For these ARs the extrapolated field lines are fitted with the corresponding AR's SXT and TRACE coronal loops. In $80 \%$ of the cases, the difference between the derived value of $\alpha_{b e s t}$ and the value of $\alpha_{b e s t}$ derived from EIT is less than $\pm 25 \%-30 \%$ of EIT's $\alpha_{\text {best }}$. More importantly, the statistical results presented earlier do not change. Furthermore in one event, vector magnetograms from Huairou Solar Observatory are available and the $\alpha_{\text {best }}$ derived from the vector magnetogram data is also consistent with the corresponding EIT's $\alpha_{\text {best }}$.

Another aspect of our study is that about $40 \%$ of our ARs (see Table 1) show coronal structures that cannot be fitted with a uniform value of $\alpha$ over the AR, indicating that the linear force-free approximation cannot represent their coronal magnetic field satisfactorily. Burnette, Canfield \& Pevtsov (2004) have argued in favor of the uniformity of the coronal value of $\alpha$ of the ARs they studied. The difference between the two studies may be due to two reasons: (1) part of our coronal dataset consists of images with better spatial resolution than the full-frame SXT images they used and (2) their dataset was dominated by mature ARs with relatively simple bipolar topologies and areas being either constant or decreasing. It is also interesting that most ARs whose coronal field deviates from the linear force-free approximation show both signs of $\alpha$ within them (see Table 1). Several such cases become obvious simply by visual inspection of the EIT images: for example in some images both S-shaped and reversed S-shaped structures appear. Such structures may correspond to positive and negative sign of $\alpha$, respectively (e.g. Rust \& Kumar). Furthermore, the change of the coronal sign of $\alpha$ is more frequent in ARs producing flares without CMEs than in those ARs producing CME-associated flares: it happens in $41 \%$ of the ARs giving flares without CMEs and only in $21 \%$ of the ARs giving CME-associated flares. Recently, Kusano et al. (2004) proposed that magnetic reconnection between oppositely sheared loops works as a trigger mechanism of solar flares. In their calculations, however, it is not clear whether the ejected flux escapes into infinity accounting for CME. Their model predicts that the position of flare brightenings should coincide with the magnetic field's shear reversals. The fact that we have used only pre-flare images, makes a direct comparison of our results with their model somewhat difficult.

\section{Conclusions and Summary}

While there is no doubt that CMEs eject helicity from the Sun, its role in the initiation of transient activity is a subject of hot debate. Some argue (e.g. Antiochos \& DeVore 1999) 
that the global helicity by itself yields little information on coronal evolution while others (e.g. Low 1996) argue that the accumulation of helicity into the corona is at the origin of CMEs. Recently, the theoretical work by Amari et al. (2003) supports that a large enough helicity seems to be a necessary condition for an ejection to occur but not a sufficient one. In this paper we investigated whether the coronal helicity has anything to do with the fact that some big flares are associated with CMEs while other big flares do not have associated CMEs. Our starting point was the dataset of big flares studied by Andrews (2003). From his dataset we selected 133 events for which suitable pre-flare photospheric magnetograms and coronal images were available. Our dataset was in a statistical sense similar to the complete Andrews's (2003) dataset because $46 \%$ of the events we analyzed did not have associated CMEs. Our computations yielded 78 ARs whose coronal magnetic field could be approximated satisfactorily under the force-free assumption and subsequently their coronal helicity was computed. From the 78 ARs, $40 \%$ produced flares without CMEs.

A key conclusion of our study is that the pre-flare coronal helicity of the ARs producing big flares that do not have CMEs is smaller, in a statistical sense, than the coronal helicity of the ARs producing CME-associated big flares. Overall, our study indicates that the amount of the stored pre-flare coronal helicity may determine whether a big flare will be a confined event (i.e. flare without CME) or an eruptive event (i.e. CME-associated flare). The findings supporting this conclusion are:

- The average values of $\alpha_{\text {best }}$ and coronal helicity are $0.035 \pm 0.018 \mathrm{Mm}^{-1}$ and $(26.8 \pm$ $18.1) \times 10^{42} \mathrm{Mx}^{2}$ for the ARs producing eruptive events but only $0.018 \pm 0.010 \mathrm{Mm}^{-1}$ and $(8.3 \pm 5.2) \times 10^{42} \mathrm{Mx}^{2}$ for the ARs producing confined events.

- About $45 \%-52 \%$ of the ARs producing confined events are associated with values of $\alpha_{\text {best }}$ and coronal helicities $H_{c}$ that are smaller than the values of $\alpha_{b e s t}$ and $H_{c}$ of all ARs producing eruptive flares.

- ARs with $\alpha_{\text {best }}>0.02 \mathrm{Mm}^{-1}$ and $H_{c}>15 \times 10^{42} \mathrm{Mx}^{2}$ are likely to produce confined flare with probabilities of only $29 \%$ and $16 \%$, respectively.

- In the ARs where the linear force-free model is not acceptable, the change of the coronal sign of $\alpha$ within an AR occurs more often in those ARs producing confined flares (in $41 \%$ of them) than in the ARs producing eruptive flares (only in $21 \%$ of them). This finding may indicate that the distribution of coronal helicity in CME-productive ARs is more coherent than in ARs giving events that do not have associated CMEs.

Finally, a word of caution is needed. Our study does not necessarily imply that the amount of coronal helicity stored in a pre-flare configuration is the only factor which determines whether the flare will be confined or eruptive. A detailed study of the pre-flare magnetic topology is also required in order to settle this issue. However, such analysis was beyond the scope of this paper and it will be carried out in the future.

\section{References}

Amari, T., Luciani, J.F., Aly, J.J., Mikic, Z., \& Linker, J. 2003, ApJ, 595, 1231

Andrews, M.D. 2003, Sol. Phys., 218, 261

Antiochos, S.K. \& DeVore, C.R. 1999, in Magnetic Helicity in Space and Laboratory Plasmas, ed. M.R. Brown, R.C. Canfield, \& A.A. Pevtsov, AGU: Geophysical Monograph 111, 187

Berger, M. A. 1985, ApJS, 59, 433

Berger, M. A. 1988, A\&A, 201, 355

Burnette, A.B., Canfield, R.C., \& Pevtsov, A.A. 2004, ApJ, 606, 565

Demoulin, P., et al. 2002, $A \& A, 382,650$

Green, L., M., Lopez Fuentes, M. C., Mandrini, C.H., Démoulin, P., van Driel-Gesztelyi, L., \& Culhane, J.L. 2002, Sol. Phys., 208, 43

Kusano, K., Maeshiro, T., Yokoyama, T., \& Sakurai, T. 2004, ApJ, 610, 537

Low, B.C. 1996, Sol. Phys., 167, 217.

Pevtsov, A.A., Canfield, R.C., \& Metcalf, T.R. 1995, ApJ, 440, L109

Rust, D.M., \& Kumar, A. 1996, ApJ, 464, L199 


\section{Discussion}

HAIShENG Ji: For a specific active region, did you check the $\alpha$ values and helicity values before and after flare/CME event?

Nindos: I didn't consider the values of $\alpha$ and helicity after the flare.

Zhukov: The appearance (or not) of a flare and/or a CME depends on the availability in a given place of free magnetic energy, which is converted into the radiated energy (flare) and/or kinetic energy (CME). As several previous speakers mentioned, the emergence of new magnetic flux is an important property of flares/CMEs, which may provide this additional free energy. My question is: what is the additional information provided by the helicity balance in comparison to the energy balance consideration?

Nindos: 1. Helicity, according to the Taylor hypothesis, dissipates much slower than magnetic energy. It is the only quantity which is conserved under reconnection.

2. Sometimes flux emergence contributes to the increase of the absolute value of the AR's helicity. But this is not always the case, as previous speakers demonstrated.

3. I don't imply that helicity is the only parameter controlling whether a flare will be eruptive or confined.

Definitely we need to study helicity and topology together.

Kоuтснму: You were considering cases of flares which did not produce CMEs. We know that there are also filament eruptive case which do not produce CMEs. The mass loading process and filament formation/eruption is an important aspect of this CME physics. Do you see any relationship between the helicity behavior with respect to the production of CMEs and the filament eruption phenomenon?

Nindos: I didn't consider the topology of the eruption. I only computed the global pre-flare coronal helicity. 